\title{
Polarity Reversal in Homologous Series of Surfactant-Free Janus Nanoparticles: Toward the Next Generation of Amphiphiles
}

\author{
Dalin $\mathrm{Wu}^{\dagger}{ }^{\dagger}$ Jia Wei Chew, ${ }^{\ddagger}, \S$ and Andrei Honciuc* ${ }^{\dagger} \dagger$ \\ ${ }^{\dagger}$ Institute of Chemistry and Biological Chemistry, Zurich University of Applied Sciences, Einsiedlerstrasse 31, 8820 Waedenswil, \\ Switzerland \\ ${ }^{\ddagger}$ School of Chemical and Biomedical Engineering, Nanyang Technological University (NTU), Singapore 637459, Singapore \\ ${ }^{\S}$ Singapore Membrane Technology Center, Nanyang Environment and Water Research Institute, Nanyang Technological University \\ (NTU), Singapore 637141, Singapore
}

\section{Supporting Information}

ABSTRACT: The ability to finely tune the amphiphilic balance of Janus nanoparticles (JNPs) could represent a step forward toward creating the next generation of solid-state amphiphiles with significant potential for applications. The inherent amphiphilicity of JNPs stemming from an intrinsic polarity contrast between two surface regions is well-acknowledged, but remained difficult to demonstrate experimentally in the absence of surfactants and stabilizers. We have designed two homologous series of surfactant-free polymeric JNPs starting from polystyrene (PS) seed nanoparticles (NPs) on which we grew Janus lobes of different sizes via seed polymerization and phase separation of the 3-(triethoxysilyl)propyl-methacrylate (3TSPM) monomer. The two series differ only by the radical initiator used in the seed polymerization: polar ionic ammonium persulfate (APS) vs nonpolar oil-soluble 2,2'-azobis(2-methylpropionitrile) (AIBN). To compare the two series, we employed them in the emulsification of water with heptane or molten paraffin wax. A polarity reversal of the JNPs within AIBN-JNP series could be observed from the catastrophic and transitional emulsion phase inversions and occurred when the more polar lobe was larger than the nonpolar seed PS lobe. Furthermore, the AIBN-JNPs appeared to be amphiphilic and adopt preferred orientation within the monolayer at the oil/water interface. We therefore demonstrated that in the absence of surfactants the amphiphilicity of the JNPs depends not only on the relative size of the lobes, but also on the surface polarity contrast, which can be tuned by changing the nature of radical initiator.

\section{INTRODUCTION}

The amphiphilic Janus nanoparticles (JNPs) resemble molecular surfactants in that they exhibit a polar/nonpolar duality. These nanoparticles, initially conceptualized by Casagrande ${ }^{1}$ followed by de Gennes, ${ }^{2}$ were named after the two-faced roman god Janus. For the last two decades, this new concept represented a meeting ground for chemists and material scientists to conjure up imaginative, nonstandard synthetic methods, resulting in an assortment of JNPs, spanning hybridized-like orbitals, ${ }^{3}$ snowman, ${ }^{4}$ mushroom shaped, ${ }^{5}$ raspberry-like, ${ }^{6}$ disk-shape, ${ }^{7}$ and so forth. Unlike the molecular surfactants, JNPs are able to carry bulklike properties, such as magnetic and optic, ${ }^{8,9}$ can exhibit fascinating stimuli-responsive properties, $^{10-13}$ and self-assemble into new reconfigurable materials. $^{14,15}$

The interfacial activity of JNPs arising from an intrinsic polarity contrast between two surface regions has been discussed theoretically in seminal publications. ${ }^{16,17}$ Unfortunately, the true interfacial activity of JNPs remained elusive experimentally in the absence of molecular surfactants, which

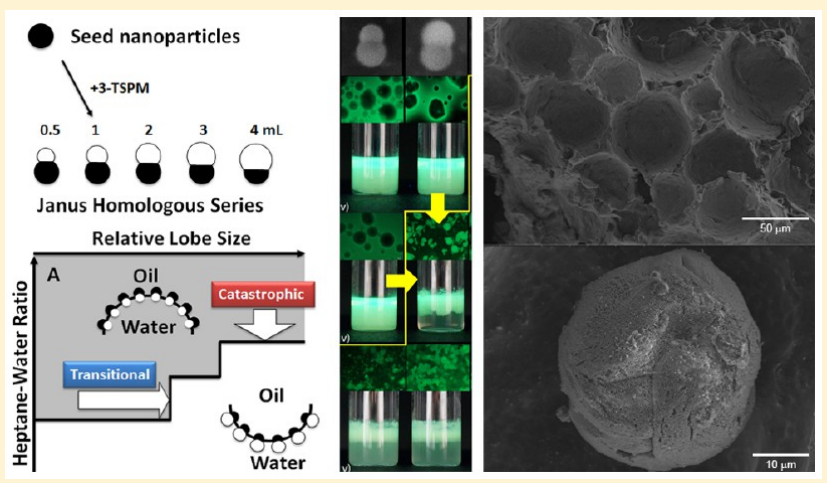

are almost always used as stabilizing agents during synthesis and are very difficult to completely remove by time-consuming serum replacement methods, such as centrifugation, dialysis, or electrodialysis. ${ }^{18-20}$ Thereby, interference from the remnant surfactants or even quenching of the JNPs' intrinsic amphiphilicity is likely. ${ }^{21}$ The synthesis of sufficient quantities of surfactant-free JNPs is necessary for understanding and harnessing the fundamental behavior of this class of materials, which forms the goal of the current effort.

To provide proof of the actual amphiphilicity of JNPs, we present the synthesis of several grams of surfactant-free poly(styrene-co-3-(triethoxysilyl)propyl-methacrylate) (PS$\mathrm{P}(3-\mathrm{TSPM})$ ) polymeric JNPs by using seed polymerization and phase separation. Furthermore, analogous to surfactants, we have created homologous series of JNPs, by using polystyrene (PS) seed nanoparticles (NPs) on which we grew

Received: April 13, 2016

Revised: June 9, 2016

Published: June 10, 2016 
the poly(3-(triethoxysilyl)propyl-methacrylate) P(3-TSPM) lobes of varying sizes as shown in Figure $1 .^{22-24}$ The polarity

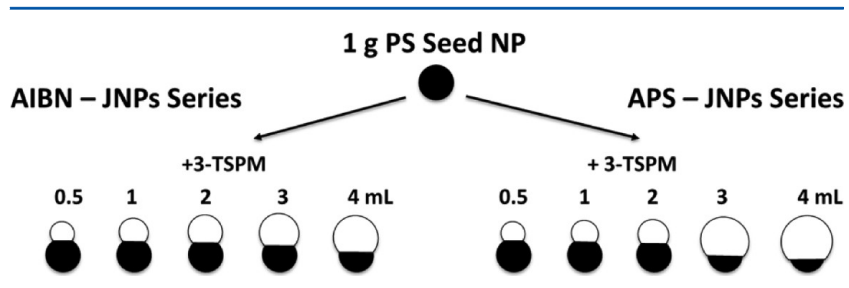

Figure 1. Cartoon representing the two homologous series of JNPs synthesized using APS and AIBN initiators from the same starting seed PS NPs. The relative lobes size of each JNP was adjusted with the amount of 3-TSPM monomer used in the reaction (namely, 0.5, 1, 2, 3 , and $4 \mathrm{~mL}$ for $1 \mathrm{~g}$ of PS seed NPs).

contrast between the Janus lobes was termed Janus balance, ${ }^{17}$ which is in principle a quantifiable parameter comparable to the hydrophilic-lyophilic balance (HLB) used for surfactants. JNPs have been successfully used to produce Pickering emulsions. The way the JNPs can assemble at the oil/water interfaces can be affected by their aspect ratio, i.e., the geometrical packing parameter and by the polarity contrast, which is, analogous to molecular surfactants, the "degree of hydrophilicity" or "degree of hydrophobicity" ${ }^{25}$ between the opposing surface regions. In this work, we also show that, in the absence of surfactant stabilizers, for a given JNP aspect ratio, the radical initiator used in the seeding-polymerization step provides means to change the surface polarity of the polymer and thus tune the native Janus balance of the JNPs. To investigate this, two homologous JNP series based on the same PS seed NPs were created in exactly the same synthetic conditions, but one with a nonionic oil-soluble radical initiator, namely, 2,2'-azobis(2-methylpropionitrile) (AIBN) and the other with an ionic water-soluble radical initiator, namely, ammonium persulfate (APS). Five JNPs of different lobe sizes were generated for each series by increasing the amount of the 3-TSPM monomer relative to $1 \mathrm{~g}$ of seed PS particles, as illustrated in Figure 1.

\section{RESULTS AND DISCUSSION}

Surfactant-Free PS Seed NPs. These were synthesized using varying amounts of divinylbenzene (DVB) via a similar procedure with the one reported by Chonde and Krieger. ${ }^{26}$ Representative scanning electron microscope (SEM) images are presented in Figure S1. The diameter of the obtained seed NPs decreased from $214 \pm 13$ to $77 \pm 10 \mathrm{~nm}$ as the amount of DVB increased from 0 to $6.75 \mathrm{~mL}$ (Table S1). The zeta potentials for all four NP batches were within the range of -49 \pm 6 to $-58 \pm 8 \mathrm{mV}$ in ultrapure water (UPW) (Table S1), all of which exceeded the colloidal stability threshold of $<-30$ $\mathrm{mV}$. For the ensuing sections, the PS seed NP used was PS2, which had a characteristic diameter of $180 \pm 10 \mathrm{~nm}$ (Table S1, in the Supporting Information).

The use of copolymerizable sodium 4-vinylbenzenesulfonate (NaVBS) surfactant monomer is essential to stabilize the monomer nanodroplets during the polymerization reaction. ${ }^{18}$ Notably, we ascertained that NaVBS was totally consumed in the copolymerization reaction, such that it was absent in the resulting solution. The surface tension of the $0.25 \mathrm{mg} / \mathrm{mL}$ PS seed NPs in UPW was $73.8 \pm 0.3 \mathrm{mN} / \mathrm{m}$ at $25{ }^{\circ} \mathrm{C}$ (Figure S2), which is close to that of pure water, indicating that the obtained PS seed NPs were surfactant-free. Furthermore, the surface tension was stable for as long as $10 \mathrm{~min}$ (Figure S2), which indicate that there was no further interfacial reorganization by surfactant detachment from the particles or "aging" effects. In addition, FTIR characterization of the seed nanoparticles showed transmittance peaks at positions 697 and $2927 \mathrm{~cm}^{-1}$ (Figure S3A), which correspond to the out-of plane bending and $\mathrm{C}-\mathrm{H}$ stretching vibration, respectively, of the phenyl rings. The appearance of a peak at $1180 \mathrm{~cm}^{-1}$ corresponding to the asymmetric vibration of the $\mathrm{SO}_{3}{ }^{-}$group demonstrates the presence of these groups in the seed NPs.

Design and Synthesis of JNPs. The second critical step in the synthesis of surfactant-free JNPs is the growth of a second lobe in the absence of surfactants by seeding polymerization from the PS seed NPs generated in step 1. For this we have used the 3-TSPM monomer, previously reported by Guignard and Lattuada, ${ }^{24}$ Sun et al., ${ }^{4}$ and Park et al. ${ }^{27}$ to be suitable for generating JNPs from a variety of seed particles via phase separation, albeit in the presence of surfactant stabilizers. The mechanisms and parameters leading to Janus particle formation from phase separation in polymer blends ${ }^{28,29}$ and phase separation by "extrusion" from seeded emulsion polymerization $^{30}$ have been previously discussed. In order to ensure polarity contrast between the JNP lobes, no surfactant copolymer was used in the second step to avoid introducing the same type of functional groups, in this case, sulfonate groups, already present in the seed NPs. Acoustic radiation was used to produce a surfactant-free oil-in-water $(\mathrm{o} / \mathrm{w})$ emulsion from the water-insoluble 3-TSPM monomer, which demonstrated good stability over several hours (Figure S4). Acoustic emulsification is a critical step to accelerate the seeding process in the absence of emulsifiers; a large number of 3-TSPM monomer droplets is needed to maximize the oil-water interfacial area and enhance monomer availability in the aqueous phase according to Kelvin-Laplace equation. ${ }^{18,31}$ Without acoustic emulsification, we were not able to obtain any JNPs in surfactant-free conditions.

Two Homologous Series of Surfactant-Free JNPs. Analogous to the homologous series of surfactants, such as linear alkyl sulfonates, or alkyl carboxylates, we have proven the possibility of creating a "homologous" series of surfactant-free amphiphilic JNPs starting from the same PS seed NPs on which we grew lobes of different sizes. Even in the absence of any surfactant emulsifiers, which were used in the method reported by Sun et al., ${ }^{4}$ acoustic emulsification of the 3-TSPM monomer allowed different sizes of these polar Janus lobes to be generated by increasing the weight ratio of 3-TSPM to PS seed NPs from 0.52 to 4.18 , which corresponds to $0.5,1,2,3$, and 4 $\mathrm{mL}$ for $1 \mathrm{~g}$ of PS seed NPs (Figure 1). For the first homologous JNP series, AIBN was used to initiate the polymerization reaction for the growth of the Janus lobe, as described in the experimental section. SEM images of the homologous series of five AIBN-JNPs clearly show that the light-gray to white $\mathrm{P}$ (3TSPM) lobe becomes progressively larger from Figures $2 \mathrm{~A}$ through E, while the darker-gray PS seed NPs (PS2, Table S1 and Figure S1B) remained approximately the same size. For ease of interpretation when referring to a particular lobe size JNPs, we adopt herein the notation "(volume of 3-TSPM monomer used) initiator type-JNPs”, e.g., (2 mL 3-TSPM) AIBN-JNPs, see Figure 1, that corresponds to approximately a 1:1 lobe aspect ratio, see Figure 2.

Moreover, the EDX spectra in Figure $2 \mathrm{~F}$, which are normalized with respect to the carbon peak at $0.28 \mathrm{keV}(\mathrm{K} \alpha)$ of the PS seed NPs, further confirms that the bulk composition of the JNPs changes in this homologous series. At this point we 

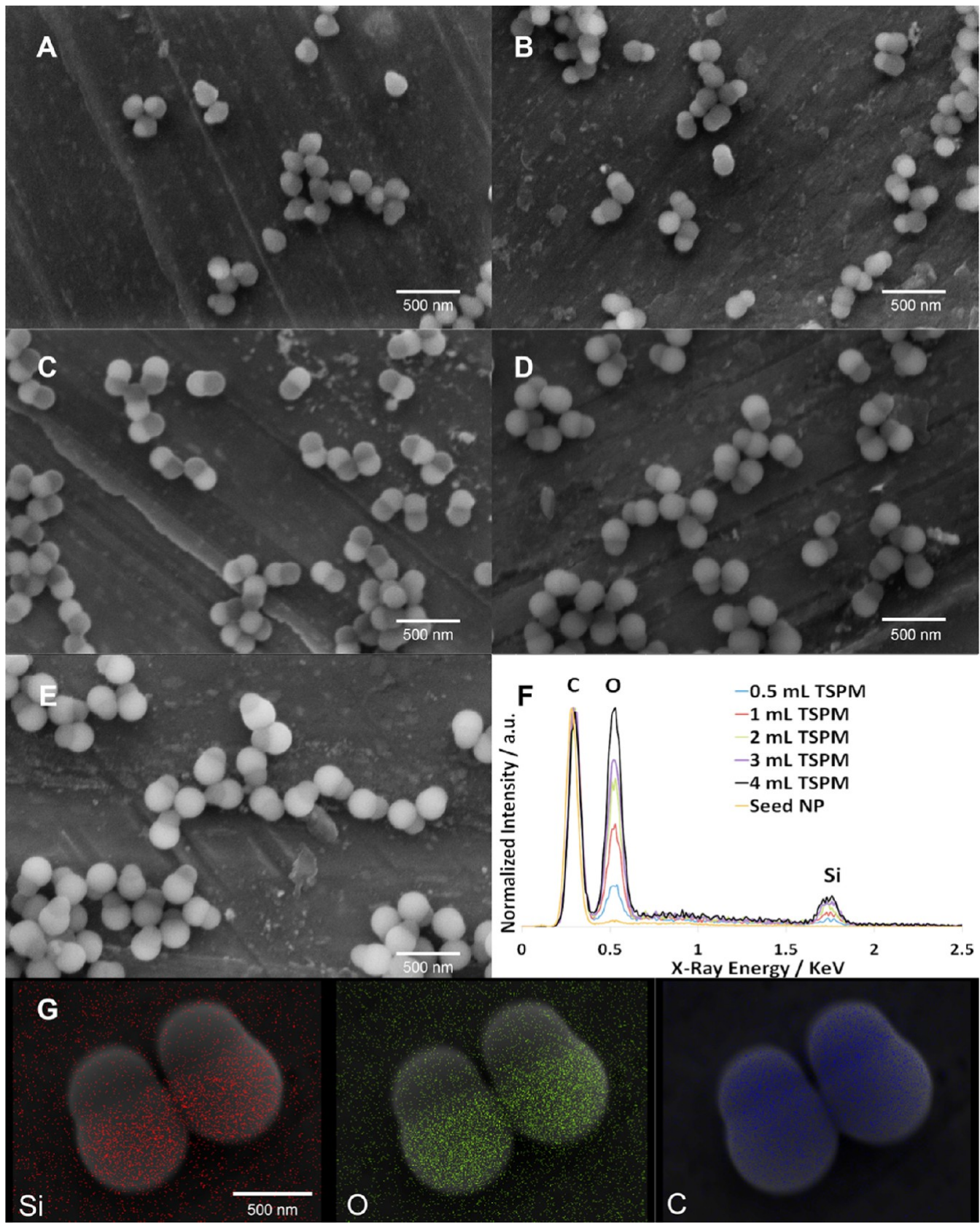

Figure 2. SEM images of AIBN-JNPs with progressively enlarged P(3-TSPM) lobe (light-gray/white) from the same seed PS NPs (dark-gray). (AE) AIBN-JNPs with progressively larger lobes obtained for a volume of 3-TSPM monomer (A) $0.5 \mathrm{~mL}$, (B) $1 \mathrm{~mL}$, (C) $2 \mathrm{~mL}$, (D) $3 \mathrm{~mL}$, and (E) 4 $\mathrm{mL}$ added to $1 \mathrm{~g}$ of PS seed NPs. (F) EDX spectra, normalized with respect to the reference carbon peak of the PS seed NPs. (G) EDX mapping of "2 mL-TSPM" JNPs obtained from larger seed PS NPs, $320 \pm 5 \mathrm{~nm}$ diameter, showing asymmetric distribution of oxygen, silicon elements, namely a higher concentration in the $\mathrm{P}$ (3-TSPM) lobe in contrast to a symmetric distribution of carbon in both Janus lobes.

emphasize that this evolution brings important information with respect to the bulk composition of the Janus lobes. The EDX spectrum corresponding to the seed PS NPs, Figure 2F "seed NP"-spectrum, shows the presence of carbon at $0.28 \mathrm{keV}$ $(\mathrm{K} \alpha)$ as expected from the monomers, styrene and DVB. With the formation of JNPs by seeded polymerization and growth of the P(3-TSPM) lobes, on the same seed PS NPs, the new elements silicon at $1.745 \mathrm{keV}(\mathrm{K} \alpha)$ and oxygen $0.52 \mathrm{keV}(\mathrm{K} \alpha)$ can be detected, seen in Figure 2F "0.5-4 mL TSPM" spectra; furthermore, their relative concentration with respect to carbon gradually increases with the increase in the P(3-TSPM) lobe size. This elemental evolution correlates directly with the increase in the light-gray to white Janus lobe, shown in the
SEM images in Figure 2A-E, which contain carbon, silicon and oxygen, while the dark-gray PS Janus lobe contains mostly carbon. The EDX spectroscopic data of the bulk JNPs is supported by the EDX mapping of the carbon, silicon and oxygen elements in individually resolved AIBN-JNPs. The EDX maps are presented in Figures $2 \mathrm{G}$ and S5, and the results clearly show that silicon and oxygen are concentrated in the $\mathrm{P}(3$ TSPM) lobe, while carbon has an even distribution between both Janus lobes. As observed in Figure S5, the EDX elemental distribution analysis was performed on larger JNPs to mitigate the issues related to this type of analysis on the smaller scales, such as lateral resolution. 


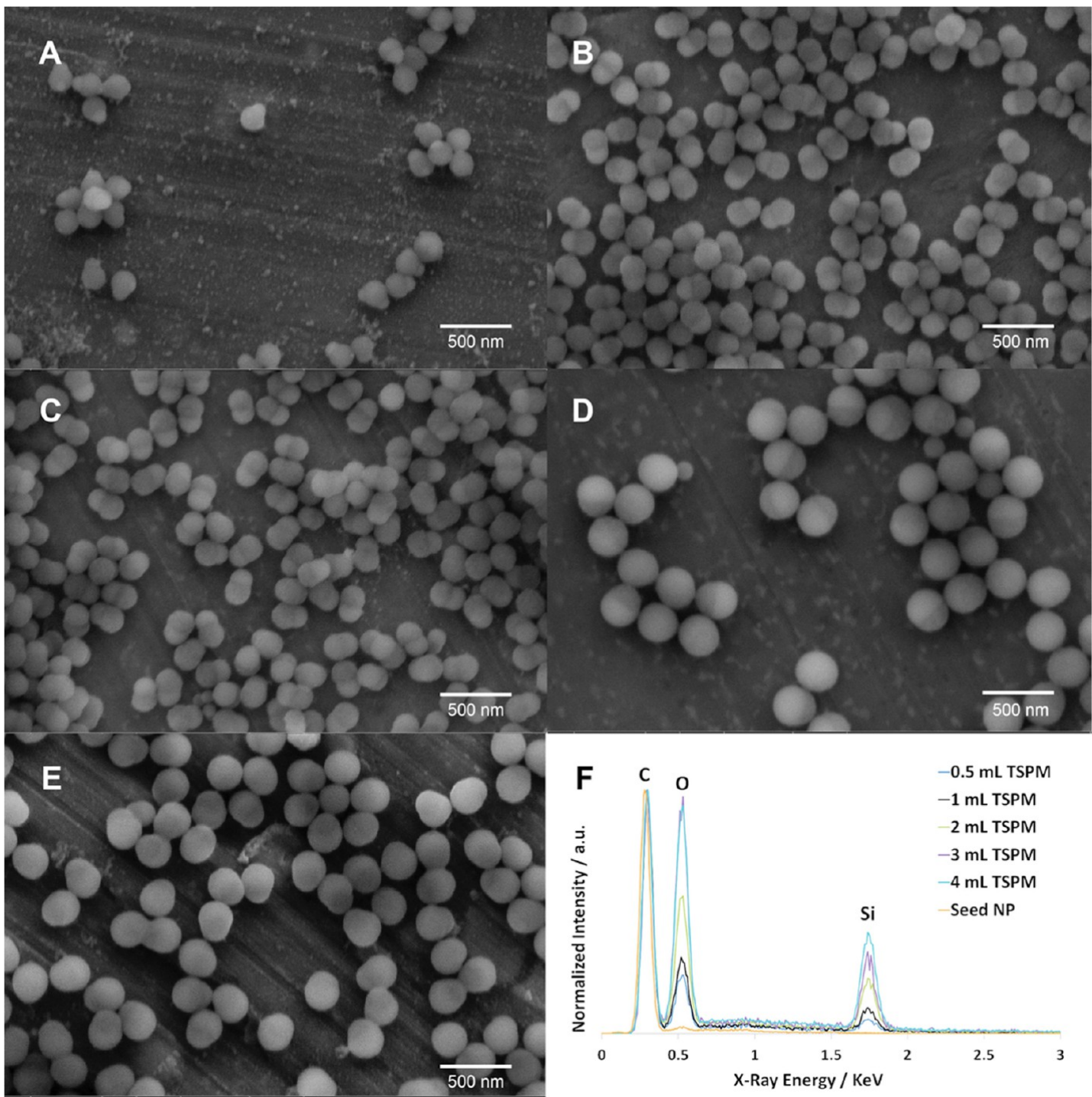

Figure 3. SEM images of APS-JNPs with progressively enlarged P(3-TSPM) lobe (lighter-gray/white) from the same seed PS NPs (dark-gray). (AE) APS-JNPs with progressively larger lobes obtained for a volume of 3-TSPM monomer (A) $0.5 \mathrm{~mL}$, (B) $1 \mathrm{~mL}$, (C) $2 \mathrm{~mL}$, (D) $3 \mathrm{~mL}$, and (E) $4 \mathrm{~mL}$ added to $1 \mathrm{~g}$ of PS seed NPs. (F) EDX spectra, normalized with respect to the reference carbon peak of the PS seed NPs.

A second homologous series of JNPs was created in the same surfactant-free condition, with the ionic water-soluble APS initiator (Figure 3). Although the exact same reaction conditions and weight ratios of 3-TSPM monomer to seed NP were used, the morphology of the APS-JNPs (Figure 3) appear different from that for AIBN-JNPs (Figure 2) in that the AIBN-JNPs assumed the more conventional snowman shape. The difference in the morphology of the AIBN-JNPs and APSJNPs is the most significant for the particles with the largest $\mathrm{P}(3-\mathrm{TSPM}$ ) lobe size (Figure $2 \mathrm{E}$ versus $3 \mathrm{E}$ ), which might indicate that the phase separation was lesser for the latter. Similarly with APS-JNPS series, the $\mathrm{Si}$ and $\mathrm{O}$ content also increased with the increase in the P(3-TSPM) lobe size (Figure 3F). The EDX analysis performed for the APS-JNPs homologous series in Figure $3 \mathrm{~F}$ shows a similar evolution with that of the AIBN-JNPs series with some differences: the larger relative intensity of the $\mathrm{Si}$ and $\mathrm{O}$ elements to that of $\mathrm{C}$ for the latter series indicates a more favorable growth of the $\mathrm{P}(3-$ TSPM) lobe when the polar initiator APS was used.

Seeded emulsion polymerization is a well-known method used to bypass the nucleation stage for obtaining a system with a precisely known number of particles; the monomer emulsion droplets act as reservoirs supplying monomers to the polymerization loci. ${ }^{32}$ Surprisingly, the polymerization results were similar, despite the use of radical initiator types of different nature (oil-soluble AIBN vs water-soluble APS) in the second step wherein the $\mathrm{P}(3-\mathrm{TSPM})$ lobes were formed, suggesting the same polymerization loci and a similar mechanism for both cases. ${ }^{30,32}$ In fact, surfactant-free emulsion polymerization studies of styrene with either APS or AIBN show that both types of initiators can drive the polymerization reaction in the aqueous phase, albeit with different kinetics. ${ }^{32,33}$ Similarly, our investigation showed that when AIBN was used to initiate the polymerization reaction, the growth of the $\mathrm{P}(3-$ TSPM) lobe appeared to be much slower than in the case of APS. Sampling of the mixtures $30 \mathrm{~min}$ from the initiation of the reaction revealed that the $\mathrm{P}(3-\mathrm{TSPM})$ lobes could readily be observed for the APS initiator, but not for the AIBN initiator (Figure S6A and C). However, the final product obtained $24 \mathrm{~h}$ after the initiation of the reaction appeared similar for both initiators (Figures S6B and D). The FTIR spectra (Figure S3A) show the appearance of at least two new peaks for the JNPs at 


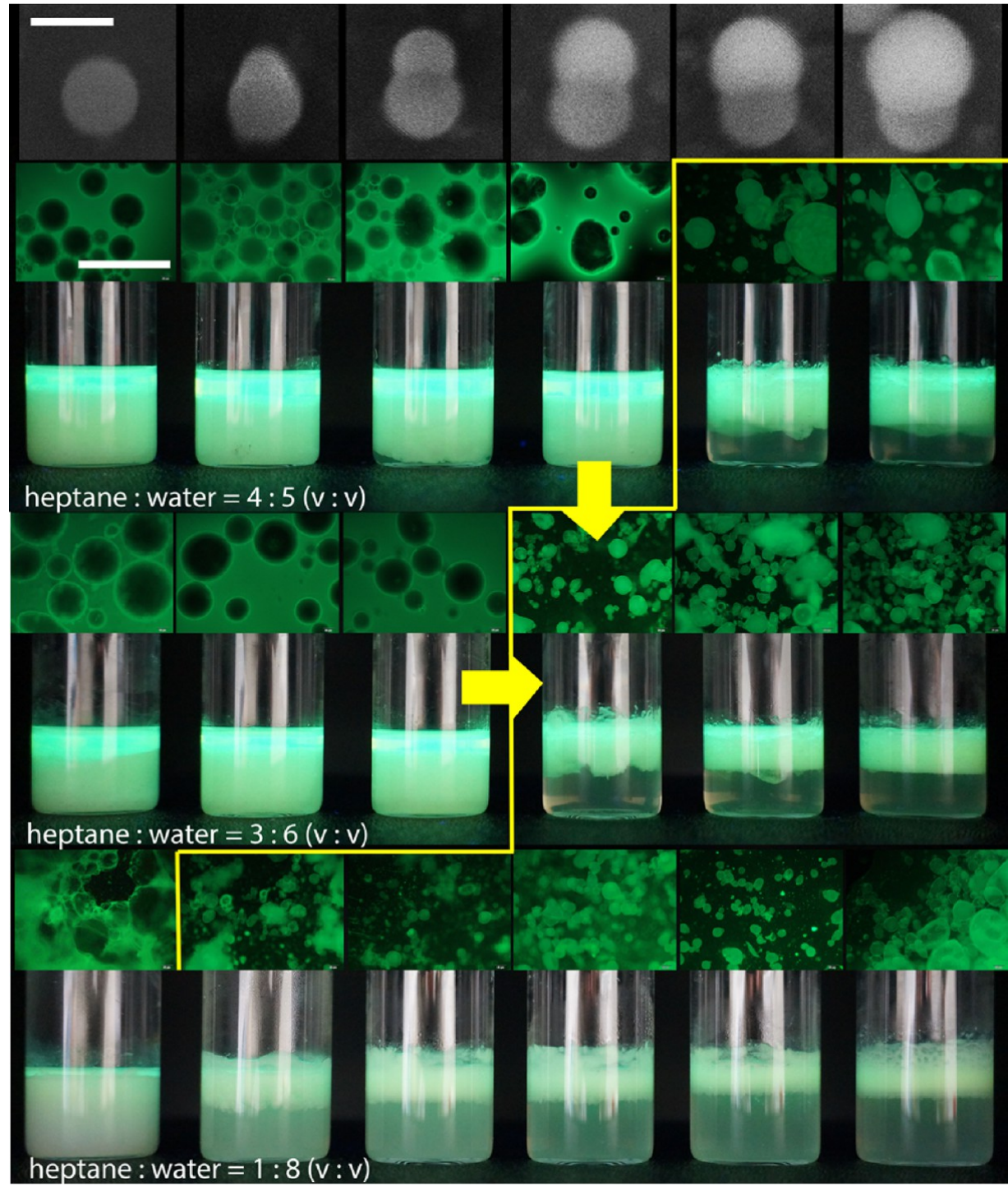

Figure 4. Formulation-composition maps with photographs of emulsions in glass vials and their corresponding fluorescence microscopy images (scale bar is $400 \mathrm{~nm}$ ) obtained with AIBN-JNPs. Top row depicts seed NPs and five AIBN-JNPs with increasing P(3-TSPM) lobe sizes (scale bar is $200 \mathrm{~nm}$ ), while the subsequent three rows represent a different volumetric ratio of heptane to water (namely, 4:5, 3:6, and 1:8), and the six columns represents the emulsification extents conferred by each type of nanoparticle. Yellow line indicates the w/o and o/w emulsion phase boundary; the vertical arrow indicates the catastrophic and the horizontal the "static" transitional phase inversion.

wavenumbers of 1719 and $1107 \mathrm{~cm}^{-1}$, which correspond to carbonyl groups and siloxane bridges $(\mathrm{Si}-\mathrm{O}-\mathrm{Si})$, respectively. ${ }^{22,23}$ Furthermore, the weak $\mathrm{O}-\mathrm{H}$ vibration typical in silanol groups at around $3462 \mathrm{~cm}^{-1}$ suggests the absence of a large amount of $\mathrm{Si}-\mathrm{OH}$ silanol groups. The zeta potential for both the homologous series of AIBN- and APS-JNPs appears to increase somewhat with the growth of the P(3-TSPM) lobe from the starting value of the PS seed NPs (Figure S3B), but no significant differences were observed between the two series.

Hydrophilic-Lyophilic Polarity Inversion in the Homologous Series of JNPs. A variety of homogeneous and asymmetric nanoparticles can be used for stabilizing emulsions and forming Pickering emulsions. Depending on the affinity to one phase or the other, oil-in-water $(\mathrm{o} / \mathrm{w})$ or waterin-oil (w/o) emulsions can be obtained, according to Finkle et al. $^{34}$ and similar to the Bancroft rules ${ }^{35}$ for surfactants. For example, carbon black particles are more likely to form w/o emulsions than the silica particles, due to their higher affinity to the nonpolar phase than to water. ${ }^{36}$ In order to gauge the change in polarity within a homologous series of five JNPs with different relative lobe sizes, the AIBN-JNPs and APS-JNPs were tested for their emulsification ability for different volumetric ratios of heptane/water mixtures, wherein heptane molecule exhibits purely nonpolar interactions. ${ }^{25}$

Photographs of the emulsions obtained with AIBN-JNPs and the corresponding fluorescence microscopy images are presented in Figure 4, whereby the top row depicts the PS seed NPs and the AIBN-JNPs with increasing lobe sizes, while the subsequent three rows represent glass vials containing emulsions obtained for three volumetric ratios of heptane: water $(4: 5,3: 6$, and $1: 8)$, and the six columns represent the emulsification extents conferred by each type of nanoparticle. More emulsification results at different solvent ratios are presented in Figure S7. Before testing the emulsification ability, the surface tension of a colloidal solution of $0.25 \mathrm{mg} / \mathrm{mL}$ of JNPs in water was determined to be $72.6 \mathrm{mN} / \mathrm{m}$ at $23{ }^{\circ} \mathrm{C}$, which is identical to that of water, suggesting that these particles were clean of surfactants (Figure S8). The time- 
dependent measurements were performed in order to monitor the potential leaching of surfactants or surface active impurities from the JNPs, but the constant value over a period of approximately $10 \mathrm{~min}$, Figure S8, clearly shows that no surface reorganization due to leaching of surfactants nor any detaching of surface adsorbed small species were detected. Although, slightly larger value of the surface tension $\sim 73.2 \mathrm{mN} / \mathrm{m}$ was recorded for the seed PS NPs, Figure S2, at the same concentration as that of JNPs, it remains within the error for this type of measurement. Here we note that a surface tension value of the colloid identical to that of pure water indicates that the JNPs do not adsorb at the air-water interface; this is presumably due to a high activation energy of attachment ${ }^{37,38}$ or surface dehydration, in contrast if this was very low, as would be the case for nonpolar particles, it would result in colloid instability and sedimentation. From a different perspective, it is noteworthy to mention the refractive index matching criterion used by Garbin et al. ${ }^{39}$ to establish whether a nanoparticle can spontaneously adsorb at interfaces, assuming that the dispersive van der Waals interaction is likely the dominating interaction between the particle and the air/water interface. In this context, the interaction energy between the JNPs and the phase 2 (air) through the intervening medium phase 1 (water) can be expressed as ${ }^{25,39} W(D)=-A_{(\mathrm{NP}) 12} \frac{R}{6 \mathrm{D}}$, where the $A_{(\mathrm{NP}) 12}$ is the corresponding Hamaker constant. Spontaneous adsorption of the particle at the interface occurs only if the Hamaker constant of the nanoparticle/water/air system remains positive, which in turn can be related through the Lifshitz's theory to the dielectric permittivity $\varepsilon_{\mathrm{i}}(i=1$-water, 2 -air, NP) and the refractive indices of refraction of the interacting media $n_{i}$, see eq $S 1$ in the Supporting Information. The Hamaker constant remains positive $A_{(\mathrm{NP}) 12}>0$, when $n_{1}$ is either larger or smaller than both indices of refraction $n_{2}$ and $n_{\mathrm{NP}}$. In this particular case, here seed PS NP or the JNP adsorption at the air/water interface, we have $n_{1 \text { (water) }}=1.33$ for water at room temperature, $n_{2 \text { (air) }}=1$ for air, and $n_{\mathrm{NP}(\mathrm{PS}-\mathrm{P}(3-\mathrm{TSPM}))}=1.4-1.6$ a range based on the available literature data on P(3-TSPM $)^{40}$ and $\mathrm{PS}^{41}$ polymers and we observe that neither of the above conditions are fulfilled for a spontaneous adsorption of the NP at the air/water interface. Therefore, it is not surprising that the colloid containing the JNPs does not show a drop in surface tension. Furthermore, if the air would be replaced with a liquid of higher index of refraction medium than that of water, e.g. heptane, $n_{1 \text { (heptane) }}=1.387$, then the NP adsorption at the liquid/liquid interface may become possible according to this criterion and also explain the formation of Pickering emulsions.

Column 1 of Figure 4 shows that the seed PS seed NPs only produced $\mathrm{w} / \mathrm{o}$ emulsion regardless of the solvent ratio due to their nonpolar nature, i.e. higher affinity to the heptane phase than water phase. Notably, both transitional and catastrophic phase inversions were observed for the homologous series of AIBN-JNPs, indicated by the yellow line in Figure 4. For the volumetric ratios of heptane: water of $4: 5$ and 3:6, a "standard" transitional phase inversions ${ }^{36}$ occurred with the increase in the size of the P(3-TSPM) lobe comparable to that of the PS lobe, i.e., aspect ratio of approximately 1:1 (horizontal arrow, Figure 4 ); whereby w/o emulsions were obtained for the JNPs possessing a $\mathrm{P}$ (3-TSPM) lobe smaller than the PS one and o/w emulsions were obtained for the JNPs with a 3-TSPM lobe equal or larger in size than the PS lobe. This transitional emulsion phase inversion can only arise from the reversal in the polarity of the particles, specifically an increase in the affinity of the JNPs toward the water phase with the increase in the $\mathrm{P}(3$ TSPM) lobe. ${ }^{36}$

Noteworthy, that the $\mathrm{P}$ (3-TSPM) lobe is more polar than the PS one can also be inferred from the emulsification data for the smallest lobe size presented in column 2 of Figures 4 and S7, for which a catastrophic phase inversion was observed, o/w emulsion was obtained for heptane: water 1:8. In contrast for the PS seed NPs only w/o emulsion could be observed for all solvent mixtures, suggesting that these have high affinity toward the oil phase and "bend" the oil-water interface toward water, or exhibits a concave tendency toward water, ${ }^{36}$ for any of the solvent mixtures tested, according to the Finkle and Bancroft rules discussed above. It can only therefore be the P(3-TSPM) lobe that increases the polarity of the JNPs from the initially nonpolar PS seeds.

The synthesis of AIBN-JNPs and the corresponding emulsification results presented in Figure 4 and S7 were repeated three times and were consistently reproducible. The fluorescence microscopy images of the emulsions in Figures 4 and S7 were taken within 2 days after their production; no significant changes were observed after 8 weeks of observation. This suggests a good stability of the AIBN-JNPs Pickering emulsions in time. After approximately 5 months, the o/w emulsions phase separated, while the w/o ones continue to remain stable.

While a catastrophic phase inversion in emulsions has been often reported for JNPs, ${ }^{4,22,36,42}$ there is no report on transitional phase inversion based on changes in the Janus balance across a homologous series of JNPs, which underscores the significance of the observation here. Furthermore, Pickering emulsions generated with $\mathrm{JNPs}^{36}$ have been studied function of particle concentration, ${ }^{5}$ solvent ratio, ${ }^{4}$ oil polarity, ${ }^{5}$ or time, ${ }^{15}$ but it remained challenging to isolate and distinguish between the contribution of the particles and that of the molecular surfactants. We hypothesize that transitional emulsion inversion in a homologous JNP series cannot be observed in the presence of molecular surfactants, which may significantly interfere with the polarity contrast or Janus balance in the JNPs and their overall effect will likely dominate due to their higher adsorption dynamics at interfaces. ${ }^{36}$

It is instructive to compare the emulsification results of the AIBN-JNPs series to those in the seminal work of $\mathrm{Tu}$ and Lee, ${ }^{12}$ whereby a "dynamic" transitional emulsion phase inversion ${ }^{36}$ was observed for a toluene: water mixture stabilized by $\mathrm{pH}$ stimuli-responsive JNPs. The increase in the $\mathrm{pH}$ value affected the relative sizes of the Janus lobes, but also the ionization degree of the $-\mathrm{COOH}$ groups and thus induced the $\mathrm{w} / \mathrm{o}$ to $\mathrm{o} / \mathrm{w}$ transition; we also note that in this case the poly(vinyl alcohol) and poly(vinylpyrrolidone) were used as interfacial stabilizers during synthesis, but were reportedly removed by multiple washing cycles. Similarly, the $\mathrm{pH}$ triphasic polymeric Janus JNPs producing transitional "dynamic" emulsion phase inversion of an initially water/dodecane to dodecane/water upon increasing the $\mathrm{pH}$ from 3 to 10 was reported by $\mathrm{Lu}$ and $\mathrm{Urban}^{43}$ in this case, the dioctylsulfosuccinate surfactant used in the synthesis of JNPs was removed by dialyzing the colloid for 3 days. However, in neither of the cases reported above, it was not explicitly shown that the molecular surfactants and stabilizers were completely removed from the colloid. On the other hand, Passas-Lagos and Schüth ${ }^{5}$ aimed specifically at obtaining a transitional phase inversion in a homologous series of $\mathrm{Fe}_{2} \mathrm{O}_{3} / \mathrm{DVB}$ (hydrophobic) $-\mathrm{SiO}_{2}$ (hydrophilic)-JNPs by increasing the $\mathrm{SiO}_{2}$ lobe size, but were 


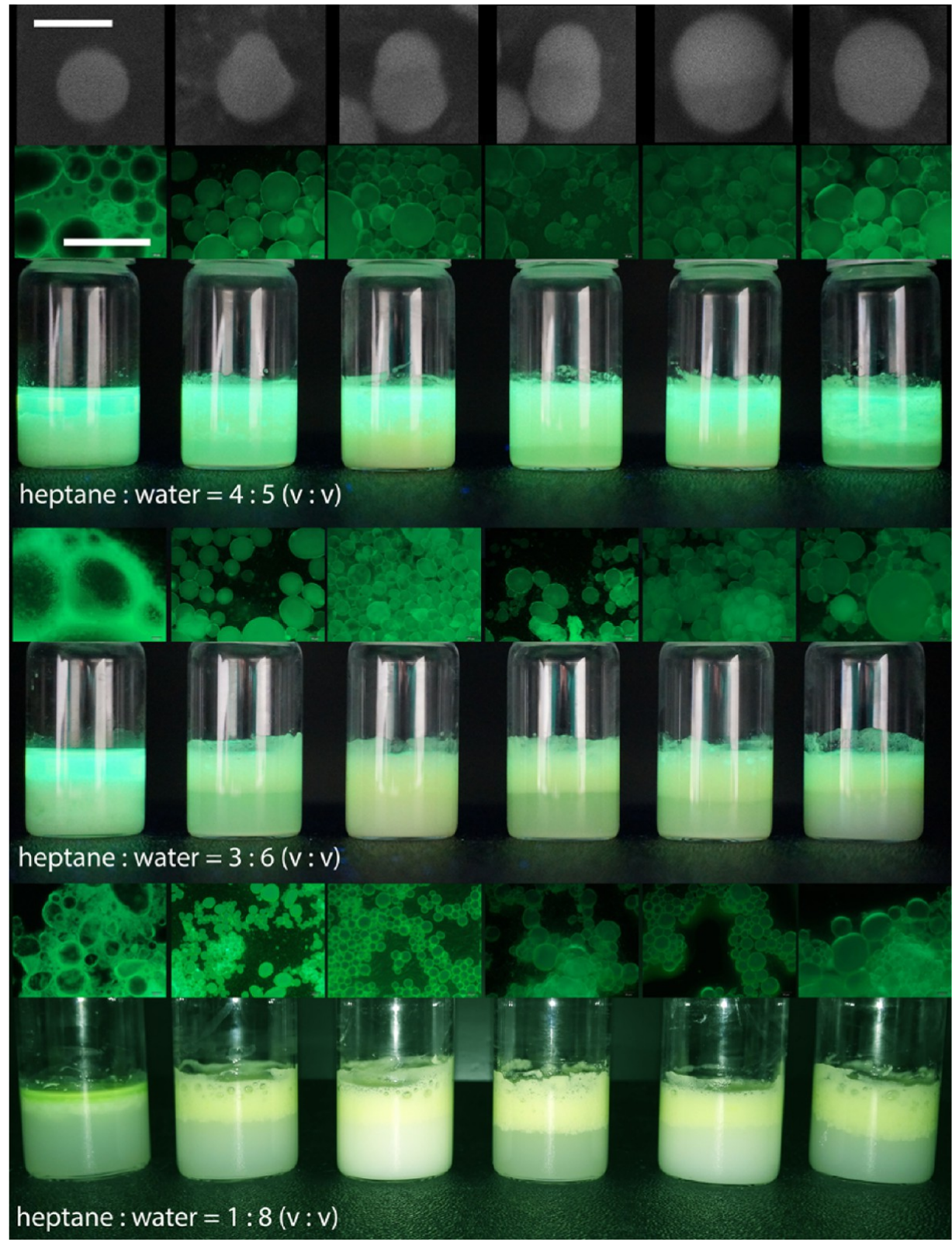

Figure 5. Formulation-composition maps with photographs of emulsions in glass vials and their corresponding fluorescence microscopy images (scale bar is $400 \mathrm{~nm}$ ) obtained with APS-JNPs. Top row depicts seed NPs and five APS-JNPs with increasing P(3-TSPM) lobe sizes (scale bar is 200 $\mathrm{nm}$ ), while the subsequent three rows represent a different volumetric ratio of heptane to water (namely, 4:5, 3:6, and 1:8), and the six columns represents the emulsification extents conferred by each type of nanoparticle.

unsuccessful in observing it for either toluene/water or vegetable oil/water mixtures.

The emulsions obtained from the second homologous series of APS-JNPs (Figures 5 and S9) show a different situation. In this case, only $\mathrm{o} / \mathrm{w}$ emulsions were obtained, regardless of either the relative lobe sizes of the JNPs or the volumetric ratio of the solvents. Notably, the qualitative difference is apparent upon comparing the fluorescence images (Figures S7B and S9B) of the o/w emulsions obtained with the AIBN-JNPs and APS-JNPs, specifically that the latter gave larger and spherical droplets while the former smaller but somewhat aspherical shaped heptane droplets. The APS-JNP synthesis (Figure 3) and the emulsification results (Figure S9) were repeated three times and were reproducible.

The evidence suggests the greater affinity of the APS-JNPs for the polar water phase than for the nonpolar heptane throughout the homologous series with no qualitative changes upon increasing of the more polar P(3-TSPM) lobe. Therefore, the difference between the behaviors of the two series can be attributed to the polarity contrast between the Janus lobes, while the aspect ratio ${ }^{25}$ (given in Tables S2 and S3) seem identical for the corresponding JNPs of each homologous series, compare Figures 2 and 3. A slight difference in the aspect ratios of JNPs between the two homologous series appears for the larger P(3-TSPM) lobes, specifically a more pronounced tendency to form core-shell structures for the last two members of the APS-JNPs series. Mock et al. ${ }^{30}$ proposed that an increased wettability of the seed particle by the second polymer may lead to core-shell NPs rather than asymmetric JNPs with well-defined lobes. In this work, the use of the APS initiator for the seed polymerization may have also changed the surface wettability and the polarity of the initially nonpolar PS 
Table 1. HLB Values and the Aspect Ratio of Janus Nanoparticles within the AIBN-JNP Series Function of the Amount of TSPM Added Relative to $1 \mathrm{~g}$ of Seed PS NPs ${ }^{a}$

\begin{tabular}{|c|c|c|c|c|}
\hline AIBN-JNPs volume of TSPM/mL & area of PS-lobe $\left(\times 1000 / \mathrm{nm}^{2}\right)^{b}$ & area of $\mathrm{P}(3$-TSPM $)$-lobe $\left(\times 1000 / \mathrm{nm}^{2}\right)^{b}$ & JNPs aspect ratio $\mathrm{P}(3-\mathrm{TSPM}): \mathrm{PS}$ & HLB number ${ }^{c}$ \\
\hline 0.5 & 81.0 & 19.5 & 0.2 & 4 \\
\hline 1.0 & 82.0 & 41.4 & 0.5 & 7 \\
\hline 2.0 & 86.7 & 79.0 & 0.9 & 10 \\
\hline 3.0 & 64.8 & 107.4 & 1.7 & 12 \\
\hline 4.0 & 55.1 & 132.7 & 2.4 & 14 \\
\hline
\end{tabular}

${ }^{a}$ We have considered the PS-lobe the non-polar lobe while the P(3-TSPM) was taken as the polar lobe. ${ }^{b}$ The areas of the lobes were calculated from the equation $A=2 \pi R h$, where $h$ is the height of the Janus lobe and $R$ is its radius, Figure S10C, and includes the average of ca. 7 particles from the corresponding SEM images presented in Figure 2. ${ }^{c}$ Value calculated with eq 1, and approximately at the same values would have arrived if the volumes of the lobes were considered instead.

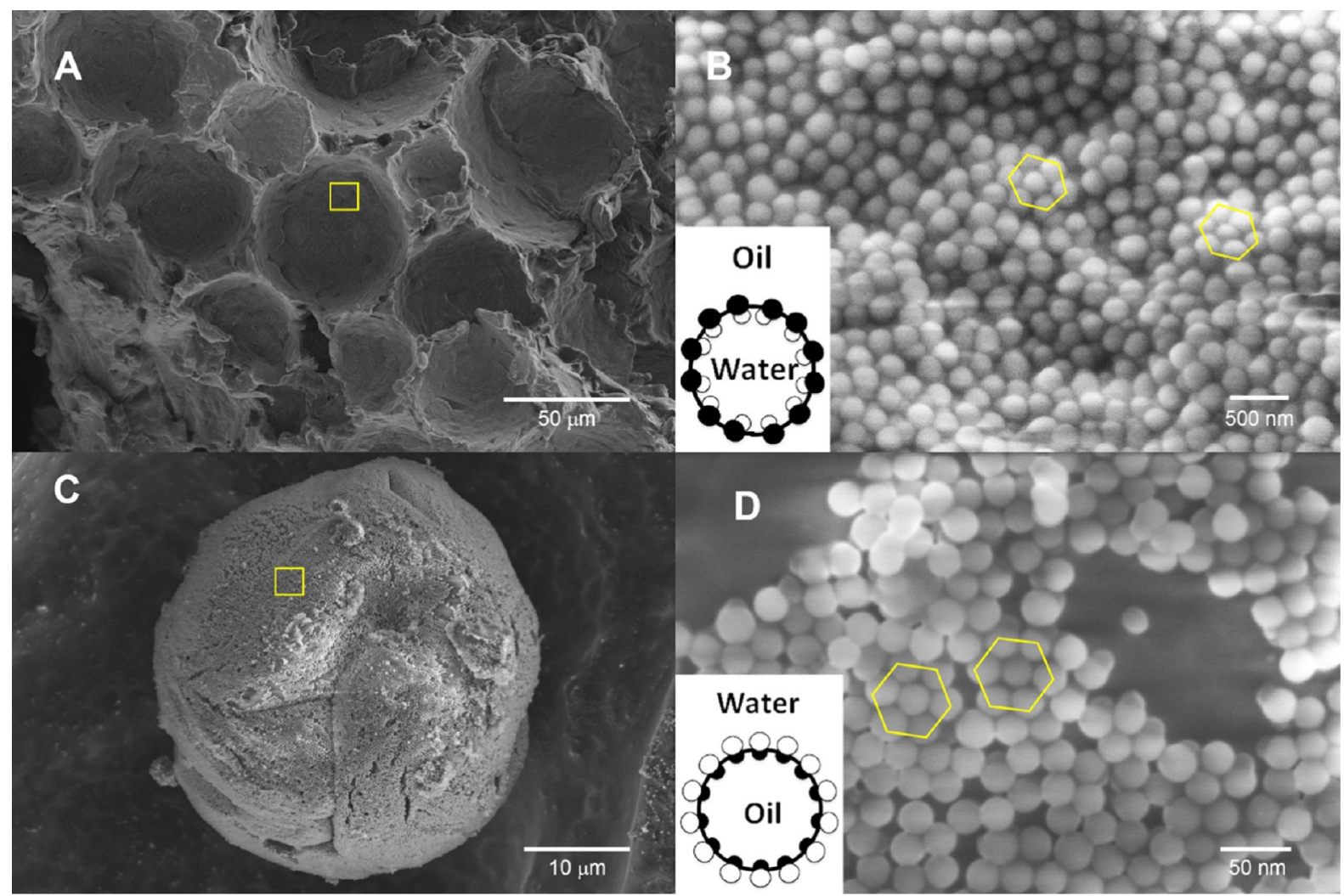

Figure 6. (A) Cross-sectional SEM image of the hollow structure resulted from cooling and solidification of the water/paraffin emulsion stabilized by the (2 mL 3-TSPM) AIBN-JNPs and (B) magnified region showing the monolayer at the water/paraffin interface where the preferential orientation of JNPs is such that PS lobe (darker) lies down toward paraffin. (C) SEM image of the paraffin colloidosome resulting from cooling and solidification of the paraffin/water emulsion stabilized by the (4 mL 3-TSPM) AIBN-JNPs and (D) magnified region showing the monolayer at the colloidosome surface, where the preferential orientation of JNPs is such that PS lobe (darker) lies down toward paraffin. Insets of (B) and (D) are cartoons depicting the type of emulsion and the orientation of the JNPs, PS (black) lobe and P(3-TSPM) (white) lobe. Only (A) and (B) samples were $\mathrm{Au}$ sputtered.

seed NPs, but this remains challenging to determine quantitatively. On the other hand, the fact that the resulting APS-JNPs are indeed more polar than the AIBN-JNPs can be deduced from the emulsification data. Furthermore, the emulsification data clearly show that the ionic nature of APS led to obtaining particles with a polar surface with a higher affinity for the water phase than for the oil even for the $(0.5 \mathrm{~mL}$ 3-TSPM) APS-JNPs, column 2 of Figure 5. This is in contrast with both the seed PS NPs whose affinity is clearly toward the oil phase and even the (0.5 mL 3-TSPM) AIBN-JNPs whose affinity is toward the oil phase. The fact that the synthetic methods are identical except the radical initiator comes to show its dramatic influence on the surface polarity and polarity contrast of the produced JNPs. It is known that the APS radical initiator introduces polar sulfate groups at the surface of polymer NPs. ${ }^{18,33}$ Hence, despite the similarity in bulk composition of the AIBN-JNPs (Figure 2F) and APS-JNPs (Figure $3 \mathrm{~F}$ ), and similar aspect ratio, it is their surface polarity leading to different emulsification capability depending on the nature of initiator used.

We have thus far established that the APS-JNPs are more polar than the AIBN-JNPs from their higher affinity toward water. Moreover, the polarity reversal observed within the AIBN-JNP homologous series implies that these are also amphiphilic and this behavior resembles that of a homologous series of molecular surfactants whose polarity reverses above and below the midrange in the HLB scale. Indeed based on the change in the relative area of the polar P(3-TSPM) lobe to the 


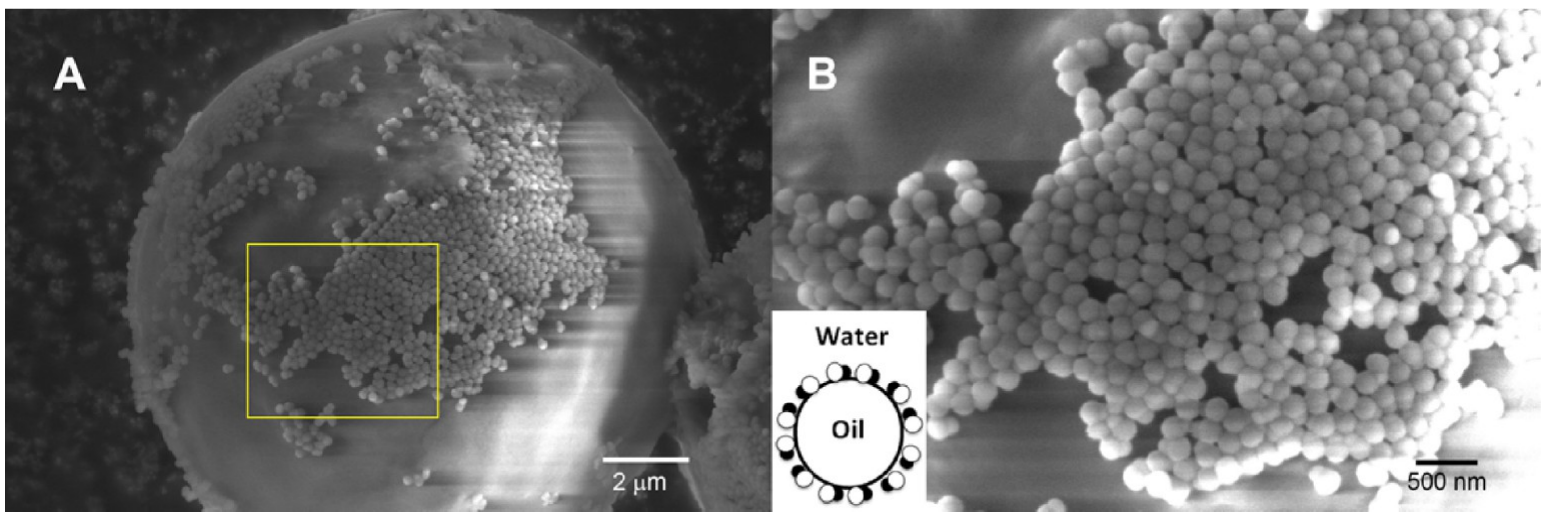

Figure 7. (A) SEM image of the paraffin colloidosome resulting from cooling and solidification of the paraffin/water emulsion stabilized by the (4 $\mathrm{mL}$ 3-TSPM) APS-JNPs and (B) magnified region showing the monolayer of JNPs at the water/paraffin interface where no preferential orientation of the JNPs can be observed instead they appear to lie flat at the interface. Inset of (B) is a cartoon depicting the type of emulsion and the APS-JNPs lying flat at the interface, PS (black) lobe and P(3-TSPM) (white) lobe.

less polar PS lobe within the AIBN-JNP series it is possible to calculate the HLB numbers for all the Janus particles in this homologous series. For this calculation, we used the following equation, resembling the earlier work of Griffin, ${ }^{44}$ which was thoroughly reviewed by Pasquali et al.: ${ }^{45}$

$$
\mathrm{HLB}=20 \frac{A_{\mathrm{P}(3-\mathrm{TSPM})} F_{1}}{A_{\mathrm{P}(3-\mathrm{TSPM})} F_{1}+A_{\mathrm{PS}} F_{2}}
$$

where the $A_{P(3-T S P M)}$ is the area of the polar lobe, $A_{P S}$ the area of the nonpolar PS lobe and in addition we have introduced $F_{i j(i=1,2)}$-weighing factors accounting for the "degree" of polarity of the lobes. The original approach of Griffin for surfactants does not account for the polarity of the surfactant moieties, but only considers their relative molecular weights: $20 \times \mathrm{M}_{\mathrm{w} \text { (polar) }} /$ $M_{\mathrm{w} \text { (molecule) }}$. The eq 1 takes the value of 20 for $F_{2}=0$ and 0 for $F_{1}=0$, which are two extreme situations: strongly polar and nonpolar particles respectively, with no amphiphilicity. On the other hand a value of $F_{1}=1$ (hypothetical $100 \%$ polar surface) and $F_{2}=1$ (hypothetical $100 \%$ nonpolar surface) assumes an "ideal" polarity contrast between the two surface regions, see Figure S10A, and thus the HLB are decided by the geometry of the lobes, i.e. their aspect ratio. The results obtained for $F_{1}=1$ and $F_{2}=1$ for the AIBN-JNPs are given in Table 1. From the calculations presented in the Table 1 it can be clearly observed that the HLB number increases when the P(3-TSPM) lobe becomes larger, thus covering almost the entire range of the HLB scale (Griffin's classification ${ }^{46}$ ) with the extreme values obtained at 4 and 14 . The fact that the members of the AIBNJNPs homologous series have HLB numbers below and above the midrange value $\sim 10$ correlates well with the emulsion phase inversion and polarity reversal observed within this series. A less arbitrary value could be assigned for $F$, and this can be calculated from the ratio between the polar and nonpolar dispersive surface energy components for each of the Janus lobes, according to the discussion in Figure $\mathrm{S} 10 \mathrm{~B}$ in the Supporting Information:

$$
F_{1}=\frac{\gamma_{\mathrm{P}(3-\mathrm{TSPM})}^{\mathrm{p}}}{\gamma_{\mathrm{P}(3-\mathrm{TSPM})}^{\mathrm{p}}+\gamma_{\mathrm{P}(3-\mathrm{TSPM})}^{\mathrm{d}}} \quad \text { and } \quad F_{2}=\frac{\gamma_{\mathrm{PS}}^{\mathrm{d}}}{\gamma_{\mathrm{PS}}^{\mathrm{p}}+\gamma_{\mathrm{PS}}^{\mathrm{d}}}
$$

where the small Greek gammas are the surface energies and the superscripts "p" and " $\mathrm{d}$ " indicate the polar and dispersive surface energy components of the corresponding Janus lobe. However, determining the surface energy of the each individual
Janus lobes is nontrivial and we are currently working in this direction and the results will be presented in a future publication.

A polarity reversal within the APS-JNP series was not observed suggesting that they are likely weakly amphiphilic, that is $F_{1} \gg F_{2}$, in which case the calculation of the HLB number will not be meaningful for this homologous series. In order to test the above hypotheses we have further investigating the APS- and AIBN-JNPs' orientation at the interface between paraffin/water solid emulsions. The choice of paraffin $\left(\mathrm{C}_{n} \mathrm{H}_{2 n+2}\right.$, $n=24-36, \mathrm{mp} 53-57^{\circ} \mathrm{C}$ ) is justified by that fact that similarly to heptane, it is a purely dispersive substance, meaning that the dispersive van der Waals component of paraffin wax is the sole contributor to the total surface energy $\left(\mathrm{J} / \mathrm{m}^{2}\right)^{47,48}$ and the cohesive energy $\left(\mathrm{J} / \mathrm{m}^{3}\right),{ }^{49}$ as expected, for the purely nonpolar linear alkanes. The emulsification was performed with molten paraffin wax at $85{ }^{\circ} \mathrm{C}$ in the presence JNPs, from both homologous series, and the emulsion phases were similar to those presented in Figures 4 and 5. The SEM images of the solid structures obtained after the cooling of the water/paraffin or paraffin/water emulsions stabilized by the AIBN-JNPs and APS-JNPs are presented in Figure 6A,B, for the $(2 \mathrm{~mL} 3$ TSPM) AIBN-JNPs (paraffin/water 4:5) and in Figure 6C,D, for the (4 mL 3-TSPM) AIBN-JNPs (paraffin/water 2:7), respectively. The SEM results clearly show that AIBN-JNPs are oriented such that the nonpolar PS lobe is oriented toward the nonpolar paraffin wax, while the more polar P(3-TSPM) lobe remains always oriented toward water. Noteworthy, is the hexagonal centered packing obtained in the interfacial monolayer for the case of the (4 mL 3-TSPM) AIBN-JNPs and this would not be possible for unless the JNPs were vertically oriented on the interface, see Figures 6D and S11C,D. On the other hand a slightly less compact and a more relaxed orientation for the ( $2 \mathrm{~mL} 3$-TSPM) AIBN-JNPs, see Figures 6B and S11A,B can be observed; nonetheless, the diameter of the particles observed from the top of the JNP monolayer does correspond to the diameter of the $\mathrm{P}(3-\mathrm{TSPM})$ lobe. This observed preferred orientation confirms that the AIBNhomologous series do possess amphiphilicity and this can be attributed to the polarity contrast between the lobes.

On the other hand, the SEM images presented in Figure 7 of the paraffin colloidosomes obtained from using the $(4 \mathrm{~mL} 3$ TSPM) APS-JNPs and paraffin/water 2:7, show that indeed these nanoparticles also stabilize the emulsion by adsorbing at 
the oil/water interface thus generating only o/w emulsions, as expected from Figure 5. On close inspection the APS-JNPs appear to have no preferential orientation in the monolayer. Similar results were found for the paraffin/water 6:3 emulsified with (4 mL TSPM) APS-JNPs; see Figure $\mathrm{S} 12$ in the Supporting Information. The lack of orientation for the APSJNPs suggests poor amphiphilicity due to a low surface polarity contrast between the two Janus lobes.

It would be instructive to compare the emulsification behavior observed for the homologous series of AIBN-JNPs to that of a homologous series of surfactants spanning the entire HLB scale, where typically the polarity reversal is observed at the midrange, from poor water-soluble, low HLB numbers $\ll 10$ (w/o emulsifiers), to higher HLB values $\gg 10$ with good water solubility (o/w emulsifiers), as presented in Table 1. Furthermore, following the assumption that the overall free energy of dehydration (or solvation) is additively composed of independent contributions from the constituent functional groups (e.g., $-\mathrm{CH}_{3}$ group, $-\mathrm{CH}_{2}-$ methylene group, and polar $-\mathrm{COOH}$ group contribute with approximately $-3.6,-1.5$, and $+25.5 \mathrm{~kJ} / \mathrm{mol}$, respectively), ${ }^{50,51}$ one could predict the length of a straight alkyl chain at which the molecular polarity switches from hydrophilic to lyophilic across a simple homologous series of surfactants. The principle of "independent surface action" often used for hydration of proteins $^{52}$ can also be applied to the large Janus amphiphiles for which presumably only the outer shell of surface functional groups play a role in the overall polarity balance, but more detailed experimental and theoretical investigations in this directions are needed. Thus, it becomes obvious that analogous to molecular surfactants, AIBN-JNP series of amphiphiles show polarity reversal and qualitatively their polarity balance can span values below and above the midrange values on the HLB scale. In the same time the APS-JNPs homologous series appears to be rather polar, placed at the top of the HLB scale, well above the midrange, due to their poor amphiphilic contrast.

\section{CONCLUSION}

Homologous series of polymeric solid-state JNPs of varying lobe sizes and aspect ratio were synthesized for the first time in surfactant-free conditions by seed polymerization and phase separation. The seed polymerization was carried with PS NPs under the monomer-starvation condition and by adjusting the volume of the 3-TSPM monomer used JNPs of varying lobe sizes were obtained. Acoustic emulsification of the 3-TSPM monomer appeared to be the critical toward eliminating the molecular surfactants from this synthetic step. Second, when similar synthetic conditions were used, but only the radical initiator was changed from the nonionic oil soluble AIBN to the ionic water-soluble APS, similar aspect ratio for both homologous series are obtained. However, differences in the emulsification of water and hexane or molten paraffin wax mixtures were observed. Polarity reversal was observed within the AIBN-JNP homologous series, while no polarity reversal was observed within the APS-JNPs. The polarity reversal within the former series was deduced from the transitional and catastrophic emulsion phase inversion, which also implies amphiphilic behavior.

The AIBN-JNPs formulation-composition maps in Figure 4 correlated with their orientation at the interface, Figure 6, such that PS-lobe orients toward oil and the P(3-TSPM) lobe toward water proved that the obtained AIBN-JNPs do possess an inherent amphiphilicity arising from the polarity contrast between the two surface regions. The JNPs polarity reversed within the AIBN-JNPs homologous series depending on the relative size of the lobes due to their change in affinity between the water and heptane phases. By analogy with the molecular surfactants, we have shown that qualitatively the amphiphilicity of the AIBN-JNPs within the homologous series appear to vary with the relative size of the Janus lobes, and their amphiphilic balance can span values below and above the midrange values on the HLB scale.

This represents a step forward toward creating the next generation of amphiphiles and emulsifiers, with additional potential use as carriers of bulklike properties or of small actives, could exceed the capabilities of molecular surfactants in new applications. Furthermore, we clearly observed amphiphilic manifestation for JNPs as large as few hundred of nanometers, thus demonstrating that the amphiphilicity can indeed be extrapolated from single molecular entities, such as surfactants, to surfactant-free solid-state JNPs.

\section{ASSOCIATED CONTENT}

\section{S Supporting Information}

The Supporting Information is available free of charge on the ACS Publications website at DOI: 10.1021/acs.langmuir.6b01422.

Experimental section; SEM images; surface tension measurements; ATR-FTIR spectra; emulsification results; SEM images(PDF)

\section{AUTHOR INFORMATION}

\section{Corresponding Author}

*E-mail: andrei.honciuc@zhaw.ch. Tel.: +41589345283.

Notes

The authors declare no competing financial interest.

\section{ACKNOWLEDGMENTS}

We are especially grateful for the financial support of Metrohm Foundation (Herisau, Switzerland) and by ZHAW via Anschubfinanzierung. We are also grateful to Mr. Yong Zen Tan for his help and contribution with the production of the initial Pickering emulsions.

\section{REFERENCES}

(1) Casagrande, C.; Fabre, P.; Raphael, E.; Veyssie, M. Janus Beads Realization and Behavior at Water Oil Interfaces. Europhys. Lett. 1989, 9 (3), 251-255.

(2) De Gennes, P. G. Soft Matter. Rev. Mod. Phys. 1992, 64 (3), 645-648.

(3) Wang, Y.; Wang, Y.; Breed, D. R.; Manoharan, V. N.; Feng, L.; Hollingsworth, A. D.; Weck, M.; Pine, D. J. Colloids with Valence and Specific Directional Bonding. Nature 2012, 491 (7422), 51-55.

(4) Sun, Y.; Liang, F.; Qu, X.; Wang, Q.; Yang, Z. Robust Reactive Janus Composite Particles of Snowman Shape. Macromolecules 2015, 48 (8), 2715-2722.

(5) Passas-Lagos, E.; Schüth, F. Amphiphilic Pickering Emulsifiers Based on Mushroom-Type Janus Particles. Langmuir 2015, 31 (28), 7749-7757.

(6) Gong, J.; Zu, X.; Li, Y.; Mu, W.; Deng, Y. Janus Particles with Tunable Coverage of Zinc Oxide Nanowires. J. Mater. Chem. 2011, 21 (7), 2067-2069.

(7) Walther, A.; André, X.; Drechsler, M.; Abetz, V.; Müller, A. H. E. Janus Discs. J. Am. Chem. Soc. 2007, 129 (19), 6187-6198.

(8) Yan, J.; Chaudhary, K.; Chul Bae, S.; Lewis, J. A.; Granick, S. Colloidal Ribbons and Rings from Janus Magnetic Rods. Nat. Commun. 2013, 4, 1516. 
(9) Nisisako, T.; Torii, T.; Takahashi, T.; Takizawa, Y. Synthesis of Monodisperse Bicolored Janus Particles with Electrical Anisotropy Using a Microfluidic Co-Flow System. Adv. Mater. 2006, 18 (9), $1152-1156$.

(10) Berger, S.; Synytska, A.; Ionov, L.; Eichhorn, K.-J.; Stamm, M. Stimuli-Responsive Bicomponent Polymer Janus Particles by "Grafting from”/“Grafting To” Approaches. Macromolecules 2008, 41 (24), 9669-9676.

(11) Cheng, L.; Zhang, G.; Zhu, L.; Chen, D.; Jiang, M. Nanoscale Tubular and Sheetlike Superstructures from Hierarchical SelfAssembly of Polymeric Janus Particles. Angew. Chem., Int. Ed. 2008, 47 (52), 10171-10174.

(12) Tu, F.; Lee, D. Shape-Changing and Amphiphilicity-Reversing Janus Particles with $\mathrm{pH}$-Responsive Surfactant Properties. J. Am. Chem. Soc. 2014, 136 (28), 9999-10006.

(13) Walther, A.; Barner-Kowollik, C.; Müller, A. H. E. Mixed, Multicompartment, or Janus Micelles? A Systematic Study of Thermoresponsive Bis-Hydrophilic Block Terpolymers. Langmuir 2010, 26 (14), 12237-12246.

(14) Chen, Q.; Whitmer, J. K.; Jiang, S.; Bae, S. C.; Luijten, E.; Granick, S. Supracolloidal Reaction Kinetics of Janus Spheres. Science 2011, 331 (6014), 199-202.

(15) Jiang, S.; Chen, Q.; Tripathy, M.; Luijten, E.; Schweizer, K. S.; Granick, S. Janus Particle Synthesis and Assembly. Adv. Mater. 2010, 22 (10), 1060-1071.

(16) Binks, B. P.; Fletcher, P. D. I. Particles Adsorbed at the OilWater Interface: A Theoretical Comparison between Spheres of Uniform Wettability and "Janus" Particles. Langmuir 2001, 17 (16), $4708-4710$.

(17) Jiang, S.; Granick, S. Janus Balance of Amphiphilic Colloidal Particles. J. Chem. Phys. 2007, 127 (16), 161102.

(18) Tzirakis, M.; Zambail, R.; Tan, Y. Z.; Chew, J. W.; Adlhart, C.; Honciuc, A. Surfactant-Free Synthesis of Sub-100 Nm Poly(styreneCo-Divinylbenzene) Nanoparticles by One-Step Ultrasonic Assisted Emulsification/Polymerization. RSC Adv. 2015, 5, 103218.

(19) Pan, M.; Yang, L.; Guan, B.; Lu, M.; Zhong, G.; Zhu, L. Soft Matter 2011, 7, 11187.

(20) Garay-Jimenez, J. C.; Young, A.; Gergeres, D.; Greenhalgh, K.; Turos, E. Methods for Purifying and Detoxifying Sodium Dodecyl Sulfate-stabilized Polyacrylate Nanoparticles. Nanomedicine 2008, 4 (2), 98-105.

(21) Jiang, S.; Granick, S. Controlling the Geometry (Janus Balance) of Amphiphilic Colloidal Particles. Langmuir 2008, 24 (6), 24382445.

(22) Tang, C.; Zhang, C.; Sun, Y.; Liang, F.; Wang, Q.; Li, J.; Qu, X.; Yang, Z. Janus Anisotropic Hybrid Particles with Tunable Size from Patchy Composite Spheres. Macromolecules 2013, 46 (1), 188-193.

(23) Hou, H.; Yu, D.; Tian, Q.; Hu, G. Preparation, Characterization, and Properties of Hollow Janus Particles with Tailored Shapes. Langmuir 2014, 30 (7), 1741-1747.

(24) Guignard, F.; Lattuada, M. Template-Assisted Synthesis of Janus Silica Nanobowls. Langmuir 2015, 31 (16), 4635-4643.

(25) Israelachvili, J. N. Intermolecular and Surface Forces, revised third ed..; Academic Press: New York, 2011.

(26) Chonde, Y.; Krieger, I. M. Emulsion Polymerization of Styrene with Ionic Comonomer in the Presence of Methanol. J. Appl. Polym. Sci. 1981, 26 (6), 1819-1827.

(27) Park, J.-G.; Forster, J. D.; Dufresne, E. R. High-Yield Synthesis of Monodisperse Dumbbell-Shaped Polymer Nanoparticles. J. Am. Chem. Soc. 2010, 132 (17), 5960-5961.

(28) Motoyoshi, K.; Tajima, A.; Higuchi, T.; Yabu, H.; Shimomura, M. Static and Dynamic Control of Phase Separation Structures in Nanoparticles of Polymer Blends. Soft Matter 2010, 6 (6), 1253.

(29) Saito, N.; Kagari, Y.; Okubo, M. Effect of Colloidal Stabilizer on the Shape of Polystyrene/Poly(methyl Methacrylate) Composite Particles Prepared in Aqueous Medium by the Solvent Evaporation Method '. Langmuir 2006, 22 (22), 9397-9402.
(30) Mock, E. B.; De Bruyn, H.; Hawkett, B. S.; Gilbert, R. G.; Zukoski, C. F. Synthesis of Anisotropic Nanoparticles by Seeded Emulsion Polymerization. Langmuir 2006, 22 (9), 4037-4043.

(31) Tadros, T. F.; Vandekerckhove, E.; Lemmens, M.; Levecke, B.; Booten, K.; Stabilization of Emulsions, Nanoemulsions and Multiple Emulsions Using Hydrophobically Modified Inulin (Polyfructose). In Emulsion Science and Technology; Tadros, T. F., Ed.; WILEY-VCH Verlag GmbH \& Co. KGaA: Weinheim, 2009; pp 57-66.

(32) Qiu, J.; Charleux, B.; Matyjaszewski, K. Controlled/Living Radical Polymerization in Aqueous Media: Homogeneous and Heterogeneous Systems. Prog. Polym. Sci. 2001, 26 (10), 2083-2134.

(33) Nazaran, P.; Tauer, K. Nucleation in Emulsion Polymerization: Another Step towards Non-Micellar Nucleation Theory. Macromol. Symp. 2007, 259 (1), 264-273.

(34) Finkle, P.; Draper, H. D.; Hildebrand, J. H. The Theory of Emulsification. J. Am. Chem. Soc. 1923, 45 (12), 2780-2788.

(35) Bancroft, W. D. Applied Colloid Chemistry, 1st ed..; McGraw-Hill Book Co.: New York, 1921.

(36) Kumar, A.; Li, S.; Cheng, C.-M.; Lee, D. Recent Developments in Phase Inversion Emulsification. Ind. Eng. Chem. Res. 2015, 54 (34), 8375-8396.

(37) Honciuc, A.; Howard, A. L.; Schwartz, D. K. Single Molecule Observations of Fatty Acid Adsorption at the Silica/Water Interface: Activation Energy of Attachment. J. Phys. Chem. C 2009, 113 (6), 2078-2081.

(38) Honciuc, A.; Baptiste, D. J.; Campbell, I. P.; Schwartz, D. K. Solvent Dependence of the Activation Energy of Attachment Determined by Single Molecule Observations of Surfactant Adsorption. Langmuir 2009, 25 (13), 7389-7392.

(39) Garbin, V.; Crocker, J. C.; Stebe, K. J. Nanoparticles at Fluid Interfaces: Exploiting Capping Ligands to Control Adsorption, Stability and Dynamics. J. Colloid Interface Sci. 2012, 387 (1), 1-11.

(40) Zajicova, V.; Exnar, P.; Stanova, I. Properties of Hybrid Coatings Based on 3-Trimethoxysilylpropylmethacrylate. Ceram.-Silik. 2011, 55 (3), 221-227.

(41) Ma, X.; Lu, J. Q.; Brock, R. S.; Jacobs, K. M.; Yang, P.; Hu, X.-H. Determination of Complex Refractive Index of Polystyrene Microspheres from 370 to 1610 Nm. Phys. Med. Biol. 2003, 48 (24), 4165.

(42) Tang, C.; Zhang, C.; Liu, J.; Qu, X.; Li, J.; Yang, Z. Large Scale Synthesis of Janus Submicrometer Sized Colloids by Seeded Emulsion Polymerization. Macromolecules 2010, 43 (11), 5114-5120.

(43) Lu, C.; Urban, M. W. Tri-Phasic Size- and Janus BalanceTunable Colloidal Nanoparticles (JNPs). ACS Macro Lett. 2014, 3 (4), 346-352.

(44) Griffin, C. W. Calculation of HLB Values of Non-Ionic Surfactants. J. Soc. Cosmet. Chem. 1955, 5, 249-257.

(45) Pasquali, R. C.; Taurozzi, M. P.; Bregni, C. Some Considerations about the Hydrophilic-lipophilic Balance System. Int. J. Pharm. 2008, 356 (1-2), 44-51.

(46) Griffing, W. C. Classification of Surface-Active Agents by HLB. J. Soc. Cosmet. Chem. 1949, 1, 311-326.

(47) Jańczuk, B.; Białopiotrowicz, T.; Wójcik, W. The Components of Surface Tension of Liquids and Their Usefulness in Determinations of Surface Free Energy of Solids. J. Colloid Interface Sci. 1989, 127 (1), $59-66$.

(48) Owens, D. K.; Wendt, R. C. Estimation of the Surface Free Energy of Polymers. J. Appl. Polym. Sci. 1969, 13 (8), 1741-1747.

(49) Hansen, C. M. Hansen Solubility Parameters: A User's Handbook, 2nd ed.; CRC Press: Boca Raton, FL, 2007.

(50) Xiang, T.; Anderson, B. D. A Computer Simulation of Functional Group Contributions to Free Energy in Water and a DPPC Lipid Bilayer. Biophys. J. 2002, 82 (4), 2052-2066.

(51) Honciuc, A.; Schwartz, D. K. Probing Hydrophobic Interactions Using Trajectories of Amphiphilic Molecules at a Hydrophobic/Water Interface. J. Am. Chem. Soc. 2009, 131 (16), 5973-5979.

(52) Ooi, T.; Oobatake, M.; Nemethy, G.; Scheraga, H. A. Accessible Surface Areas as a Measure of the Thermodynamic Parameters of Hydration of Peptides. Proc. Natl. Acad. Sci. U. S. A. 1987, 84 (10), 3086-3090. 